\title{
The Result Analysis of Drug Susceptibility Test in Vitro of
}

\author{
Mycoplasma in Urogenital Tract \\ Yan Zhu ${ }^{a^{*}}$, Jiao Feng ${ }^{a}$, and Qingyang Liu ${ }^{b}$ \\ ${ }^{a}$ Liaoning economic management cadre Institute, China \\ b The Affiliated Hospital of Liaoning University of Traditional Chinese Medicine, \\ China \\ *Corresponding author: Yan Zhu, Master, 46581081@qq.com
}

\begin{abstract}
Purposes: To discuss the infected status and drug resistance of mycoplasma in urogenital. Methods: Five hundred and seventy-nine genitourinary samples were detected for the presence of mycoplasma with identification and 10 drug sensibilities

by Mycoplasma Susceptibility Test Kit.

Results: Two hundred and sixty-three strains (45.42\%) of mycoplasma were detected from the 579 specimens. One hundred and eighty-five samples of Uu (31.95\%), 15 samples of Mh (2.59\%) and 63 samples of $\mathrm{Uu}$ and $\mathrm{Mh}$ (10.88\%) were identified to present with positive. Because of the differences among mycoplasma types, the drug resistance of Mycoplasma to 10 antibiotics was also different.

Conclusions: Josamycin (JOS) and doxycycline (DOX) were the most sensitive drug against mycoplasma infection that, they were the first choice. Furthermore, both roxithromycin and azithromycin showed the resistance to mycoplasma infection, so they couldn't be used for treating the infection. There were some differences between drug sensitivity and drug resistance. With respect to drug susceptibility test, antimicrobial agents should be selected so as to reduce the resistant strains and give patients standard treatment.
\end{abstract}

Key words: mycoplasma; ureaplasma urealyticum (Uu); mycoplasma hominis (Mh); drug susceptibility test; drug resistance; urogenital tract

\section{Introduction}

Mycoplasma is a kind of cell without cytoderm. It is a prokaryote with simple structure and small size that is between bacteria and viruses. It has affinities with urogenital tract and respiratory mucosa. $\mathrm{Uu}$ and $\mathrm{Mh}$ is the most common kind of mycoplasma in urogenital infection (X.H. Wang et al. ${ }^{1}$ ). They often cause infectious diseases such as Nongonococcal urethritis (NGU), sterility of men and women, chronic prostatitis, salpingitis, vulvovaginitis, pelvic inflammation, etc $\left(X . M i n u^{2}\right)$.

In recent years, urogenital infections which caused by mycoplasma was increased, with a growing number of drug-resistant strains (B.Farkas et al. ${ }^{3-4}$ ). Because of the different rates of mycoplasma infection in different regions, the same 
strain had significant difference in the drug resistance rate of the same strain to the same antibiotics at different time (M.X. Qiu et $a l^{5}{ }^{5}$ ). How to select antibiotics according to the results of drug sensitivity and inhibit the drug-resistant has become the key to clinical treatment.

In order to promote rational clinical use of drug, the results of drug resistance were collected from 579 specimens by connecting with the practical situation. The detection rate of $\mathrm{Uu}$ and drug sensitivity of both $\mathrm{Mh}$ and mix groups were analyzed. Drug sensitivity was summarized. The transformation of antimicrobial susceptibility of different mycoplasma was comprehended. Drug resistance status of mycoplasma were analyzed. Therefore, the information for rational clinical use of drug were provided. Uu and Mh from 579 female urogenital tracts were detected. In addition, drug susceptibility tests were conducted and the results were analyzed. The resistances and sensitivities of josamycin (JOS), roxithromycin (ROX), minocycline (MIN), doxycycline (DOX), ciprofloxacin (CPFX), azithromycin (AZI), spectinomycin (SPE), clarithromycin (CLA), sparfloxacin (SPA) and ofloxacin (OFL) were comprehended. Information for rational clinical use of drug were provided to improve cure rate and prevent recurrence by connecting with the practical situation.

\section{Materials and methods}

\subsection{General information}

Five hundred and seventy-nine diagnosed NGU female were come from department of urology or gynecology. Their ages were between 30 and 60. Any antibiotics were unused before specimens for 1 week.

\subsection{Draw materials}

Cervix was expanded with vagina dilator. Mucus acquired from cervical duct was wiped with swabs first. Then another swab was dived into cervix uteri. It rotated for $10 \sim 30$ s in order to get a few columnar cells and the samples should be sent for inspection immediately.

\subsection{Detection methods}

The types of mycoplasma from specimens were identified with the Mycoplasma Susceptibility Test Kit (Zhuhai Yinke medical engineering limited company). Ten antibiotics sensibilities were also detected. The experiments were operated strictly according to the instruction.

\section{Results}

\subsection{Identification of mycoplasma type}

Two hundred and sixty-three strains (45.42\%) of mycoplasma were detected from the 579 specimens. One hundred and eighty-five samples of Uu (31.95\%), 15 samples of Mh (2.59\%) and 63 samples of Uu and Mh (10.88\%) were identified to present with positive. 


\subsection{Drug susceptibility test of mycoplasma}

\subsubsection{Results of drug susceptibility test of $U u$}

Three antibiotics had high sensitivities for $185 \mathrm{Uu}$ specimens. They were JOS (98.91\%), DOX (96.22\%) and MIN (94.58\%). CPFX had the highest resistance rate $(81.08 \%)$ and the others had low resistance rate $(<40.00 \%)$ (Table 1$)$.

Table 1 - Results of drug susceptibility test from 185 Uu specimens

\begin{tabular}{|l|c|c|c|c|}
\hline Antibiotics & $\begin{array}{c}\text { Number of } \\
\text { sensitivity }\end{array}$ & $\begin{array}{c}\text { Sensitive } \\
\text { rate (\%) }\end{array}$ & $\begin{array}{c}\text { Number } \\
\text { of } \\
\text { resistance }\end{array}$ & $\begin{array}{c}\text { Resistance } \\
\text { rate (\%) }\end{array}$ \\
\hline CLA & 154 & 83.24 & 8 & 4.32 \\
\hline CPFX & 1 & 0.54 & 150 & 81.08 \\
\hline DOX & 178 & 96.22 & 7 & 3.78 \\
\hline JOS & 183 & 98.91 & 1 & 0.54 \\
\hline MIN & 175 & 94.58 & 7 & 3.78 \\
\hline OFL & 24 & 12.97 & 59 & 31.89 \\
\hline ROX & 81 & 43.78 & 44 & 23.78 \\
\hline SPA & 95 & 51.45 & 44 & 24.02 \\
\hline SPE & 101 & 54.90 & 45 & 24.04 \\
\hline AZI & 118 & 63.78 & 15 & 8.11 \\
\hline
\end{tabular}

\subsubsection{Results of drug susceptibility test of $M h$}

Three antibiotics had the highest sensitivity for 15 Mh specimens. They were JOS (100.00\%), DOX (100.00\%) and MIN (100.00\%). ROX had the highest resistance rate $(86.67 \%)$, followed by AZI (80.00\%). The third highest resistance rate was occupied by CLA (66.67\%), CPFX (66.67\%), OFL (66.67\%) (Table 2).

\subsubsection{Results of drug susceptibility test of Uu and Mh}

JOS (88.88\%) had the highest sensitivity for $63 \mathrm{Uu}$ and Mh specimens followed by DOX (82.53\%), the others had low sensitivity (<30.00\%). The highest resistance rate was occupied by ROX (92.25\%), followed by AZI (92.08\%), then CPFX (92.08\%) (Table 3).

\section{Discussions}

Mycoplasma lives on urogenital tract and bases on the infection of $\mathrm{Uu}$ and Mh. Its main transmission routes are sexual transmission and mother-to-child transmission (M.X. Qiu et al. ${ }^{5}$ ). During the recent years, more and more females were suffering from infectious diseases of urogenital tract due to anatomy of the urinary tract and luminal fluid environment of the female reproductive tract. 
Table 2 - Results of drug susceptibility test from 15 Mh specimens

\begin{tabular}{|l|c|c|c|c|}
\hline Antibiotics & $\begin{array}{c}\text { Number of } \\
\text { sensitivity }\end{array}$ & $\begin{array}{c}\text { Sensitive } \\
\text { rate (\%) }\end{array}$ & $\begin{array}{c}\text { Number of } \\
\text { resistance }\end{array}$ & $\begin{array}{c}\text { Resistance } \\
\text { rate (\%) }\end{array}$ \\
\hline CLA & 3 & 20.00 & 10 & 66.67 \\
\hline CPFX & 3 & 20.00 & 10 & 66.67 \\
\hline DOX & 15 & 100.00 & 0 & 0.00 \\
\hline JOS & 15 & 100.00 & 0 & 0.00 \\
\hline MIN & 15 & 100.00 & 0 & 0.00 \\
\hline OFL & 3 & 20.00 & 10 & 66.67 \\
\hline ROX & 0 & 0.00 & 13 & 86.67 \\
\hline SPA & 9 & 60 & 2 & 13.33 \\
\hline SPE & 3 & 20.00 & 12 & 80.00 \\
\hline AZI & 10 & & & 13.33 \\
\hline
\end{tabular}

Table 3 - Results of drug susceptibility test from $63 \mathrm{Uu}$ and Mh specimens

\begin{tabular}{|l|c|c|c|c|}
\hline Antibiotics & $\begin{array}{c}\text { Number of } \\
\text { sensitivity }\end{array}$ & $\begin{array}{c}\text { Sensitive } \\
\text { rate (\%) }\end{array}$ & $\begin{array}{c}\text { Number } \\
\text { of } \\
\text { resistance }\end{array}$ & $\begin{array}{c}\text { Resistance } \\
\text { rate (\%) }\end{array}$ \\
\hline CLA & 4 & 6.34 & 53 & 84.12 \\
\hline CPFX & 1 & 1.58 & 58 & 92.08 \\
\hline DOX & 52 & 82.53 & 10 & 15.87 \\
\hline JOS & 56 & 88.88 & 5 & 7.95 \\
\hline MIN & 0 & 0.00 & 54 & 85.72 \\
\hline OFL & 2 & 3.17 & 50 & 79.37 \\
\hline ROX & 1 & 1.58 & 60 & 95.25 \\
\hline SPA & 16 & 25.39 & 38 & 60.33 \\
\hline SPE & 11 & 17.46 & 36 & 57.15 \\
\hline AZI & 1 & 1.58 & 58 & 92.08 \\
\hline
\end{tabular}

In the past few years, due to the abuse of broad-spectrum antibiotics and substandard treatment, an increasing number of drug-resistant strains of mycoplasma was emerged. Relapse or persistent of NGU was caused by mycoplasmain fection which were resistant to multiple drugs. Great difficulties were brought in clinical treatment (Q.S. Lai et al. ${ }^{6}$ ). Because the mycoplasma infection symptoms are not obvious, the treatment is usually incomplete and leads to repeated attacks. Therefore, except for rational antibacterials, enough time should be given in order to prevent recurrence (Z.G. Huang et al. ${ }^{7}$ ).

In addition, two hundred and sixty-three strains (45.42\%) of mycoplasma were detected from the 579 specimens. One hundred and eighty-five samples of $\mathrm{Uu}$ 
(31.95\%), 15 samples of $\mathrm{Mh}$ (2.59\%) and 63 samples of $\mathrm{Uu}$ and $\mathrm{Mh}$ (10.88\%) were identified to present with positive. Thus it could be seen that most of mycoplasma infection from urogenital tract were $\mathrm{Uu}$, $\mathrm{Mh}$ was the least. It was similar to many related reports (H. Qiang et al. ${ }^{8-9}$ ). The infection rate of Uu was highest because its apical structure could adhere to the surface of target cells. Moreover, its degradation products could increase vaginal $\mathrm{pH}$. It was benefit for other bacteria to multiply and infect (F.H. Liu et al. ${ }^{10}$ ).

Mycoplasma has no cell walls, so it could resistant to $\beta$-lactam antibiotics. So it is treated by disrupted protein-synthesis drugs, such as Tetracyclines, Quinolones, Macrolides, etc.

Antimicrobial resistance continued to grow due to the high infection rate of mycoplasma. A high focus would be placed on choosing the correct antibiotics. It indicated from Table 1 to 3 that, JOS, DOX and MIN had high sensitivity of Uu order. It was mainly resistant to CPFX. Mh had the same sensitive antibiotics with Uu, but it was resistant to ROX and AZM. The preceding two locations of the antibiotics which had high sensitivity of Uu and Mh were JOS and DOX. They were resistant to ROX, CPFX and AZM. In conclusion, mycoplasma infection was most sensitive to JOS and DOX. JOS is an uninducible Macrolides. It has broad spectrum antimicrobial and is effective to mycoplasma (X.L. Zhang et al. ${ }^{11}$ ). According to the results, all infections were sensitive to JOS and DOX, and were resistant to ROX and AZM. It was consistent with the results reported at home and abroad (W.M. You et al. ${ }^{12-14}$ ). Drugs with high resistance rate $(>17.0 \%)$ were genarally used for long times and repeated used. This might the major factor of drug resistance. Moreover, it might be related to irregularity and long-time use, inadequate dosage, insufficient treatment, spread of drug-resistant strains and co-infection (Y.F. Chen et al. ${ }^{15}$ ). It suggests that the proper drug selection according to the results of drug sensitivity and the pharmacokinetics of drugs in vivo was helpful to improve the clinical effect.

\section{References}

1. X.H. Wang, H. Li and C.Z. Xiong, Analysis and countermeasure of genital chlamydia trachomatis and mycoplasma infections in urogenital tract from 581cases, J. Chinese Journal of Nosocomiology. 17 (2007) 752-754.

2. X.M. Wu, Analysis of mycoplasma infection of urogenital tract and result of drug susceptibility test, J. Journal of Medical Forum. 30 (2009) 90-91.

3. B.Farkas, E. Ostorhi, K. Pnyai, et al, Frequency and anti-biotic resistance of Ureaplasma urealyticum and mycoplasma hominis in genital samples of sexually active individuals, J. OrvHetil. 152 (2011) 1698-1702.

4. Y.Zhou, X.L. Xu, C.P. Wang, M. Zhou, et al, Detection and the antibiotic susceptibility analysis of mycoplasma and chlamydia in urogenital tract infections of 327 cases patients with tubal infertility, J. Chinese Journal of Experimental and Clinical Virology, 25 (2011) 201-204.

5. M.X. Qiu, W.H. Ma, L.M. Cheng, et al. Mycoplasma infection and comparison of result of drug susceptibility test from Guangdong Puning in 2001and 2003, J. Southern China Journal of Dermato-Venereology. 11 (2004) 248-249. 
6. Q.S. Lai, M.X. Qiu and L.K. Lin, The clinical analysis of drug susceptibility test in vitro of ureaplasma urealyticum, J. China Practical Medicine. 4 (2009) 144-145.

7. Z.G. Huang, C. Huang, H.P. hong, et al. The current situation of mycoplasma infection in urogenital tract and activity study of antibacterial agents, J. International Journal of Laboratory Medicine. 29 (2008) 540-541.

8. H. Qiang, Y. Zhou, Cultivation of mycoplasma infection in urogenital and analysis of drug susceptibility test. J. Chinese Journal of Nosocomiology. 17 (2007) 107-109.

9. Z.L. Chen, K.L. Luo, Y. Liu, et al. Mycoplasma infection in female urogenital tract and analysis of drug susceptibility test. J. International Journal of Laboratory Medicine. 32 (2011) 599-600.

10. F.H. Liu, Mycoplasma infection in urogenital tract and analysis of drug susceptibility test from 224 cases. J. Practical Preventive Medicine. 15 (2008) 570-571.

11. X.L. Zhang, X.H. Fang, The result analysis of drug susceptibility test of ureaplasma urealyticum, J. International Journal of Laboratory Medicine. 12 (2009) 1219.

12. W.M. You, Y.H. Pan and P.P. Lin, The detection of mycoplasma in urogenital tract and analysis of drug resistance, J. Acta Universitatis Medicinalis Nanjing (natural science). 27 (2007) 268-271.

13. J. Zhan, Z.X. Lu, The detection of mycoplasma in urogenital tract and analysis of drug susceptibility test, J. International Journal of Laboratory Medicine. 31 (2010) 605-606.

14. S.Pereyre, H. Renaudin, A. Charron, et al. Emergence of a 23SrRNA mutation in Mycoplasma hominis associated with a loss of the intrinsic resistance to erythromycin and azithromycin, J. J Antimicrob Chemother. 57 (2006) 753-756.

15. Y.F. Chen, K. Zou, MycoplasmaCultureandAnalysisof SusceptibilityTest, J. Chinese Journal of Nosocomiology. 19 (2009): 2208-2209. 